\title{
A Multiplex PCR Assay for Six Aedini Species, Including Aedes albopictus
}

\author{
Woo Jun Bang \\ Kyungpook National University College of Natural Sciences \\ Min Hyeok Won \\ Kyungpook National University College of Natural Sciences \\ Seong Tae Cho \\ Kyungpook National University College of Natural Sciences \\ Jihun Ryu \\ Kyungpook National University College of Natural Sciences \\ Kwang Shik Choi ( $\nabla$ ksc@knu.ac.kr) \\ Kyungpook National University, Daehak-ro 80, Daegu
}

\section{Methodology}

Keywords: Aedini species, Aedes albopictus, Internal transcribed spacer 2(ITS2), multiplex PCR assay

Posted Date: December 15th, 2020

DOI: https://doi.org/10.21203/rs.3.rs-127286/v1

License: () (7) This work is licensed under a Creative Commons Attribution 4.0 International License. Read Full License

Version of Record: A version of this preprint was published at Parasites \& Vectors on July 28th, 2021. See the published version at https://doi.org/10.1186/s13071-021-04871-7. 


\section{Abstract}

\section{Background}

Mosquitoes, as vectors of various human pathogens, are significant drivers of serious human illness. In particular, those species in the Aedini tribe, which typically transmit dengue virus, Chikungunya fever virus, and Zika virus, are increasing their range because of climate change and international commerce. In order to prevent mosquito-borne disease, accurate mosquito species identification and monitoring are needed. The goal of this work was to develop a rapid and simple molecular diagnostic method for six morphologically similar Aedini species (Aedes flavopictus, Aedes albopictus, Ochlerotatus koreicus, Ochlerotatus japonicus, Ochlerotatus togoi, and Ochlerotatus hatorii) in Korea.

\section{Methods}

In total, 109 samples were used in this study. The internal transcribed spacer 2 (ITS2) regions from all six species were amplified, sequenced and analyzed using Mega 6. Once regions that were consistently different in sequence between all six species were identified, multiplex primers were designed to amplify them to generate species-specific fragments distinguishable by their size.

\section{Results}

Uniquely sized fragments were generated in Aedes flavopictus (495bp), Aedes albopictus (438bp), Ochlerotatus koreicus (361bp), Ochlerotatus togoi (283bp), Ochlerotatus hatorii (220bp), and Ochlerotatus japonicus (160bp). Pairwise distance analysis showed that the difference was $35.0 \pm 1.5 \%$ between Aedes spp. and Ochlerotatus spp., $17.4 \pm 0.2 \%$ between Aedes albopictus and Aedes flavopictus, and $11.1 \pm 0.3 \%$ between Ochlerotatus koreicus and Ochlerotatus japonicus.

\section{Conclusions}

In this study, a multiplex PCR assay for six species of the Aedini tribe was developed. This assay is more accurate than morphological identification and will be useful for monitoring and controlling these vector mosquitoes.

\section{Background}

Mosquitoes are insects which are vectors for diseases such as malaria, Japanese encephalitis, and dengue virus. The threat of mosquito-borne diseases is increasing in parallel with expansion of their mosquito vector ranges. Mosquito ranges are expanding because of climate change and increased introductions into novel territory facilitated by international travel and commerce (Reiter 2001; Tatem et al., 2012). More than 250 million people are exposed to mosquitoborne dengue viruses, and more than 400,000 peoples die from malaria each year (WHO, 2020).

Among the Aedini tribe, Aedes sp. and Ochlerotatus sp. are known to transmit dengue virus, Chikungunya virus, and yellow fever virus, and have been recently shown to be the main vector of Zika virus (Bhatt et al., 2013; Attaway et al., 2016). The main species that serve as vectors for these diseases are Aedes aegypti and Aedes albopictus. Notably, the range of Ae. albopictus, which is a potential vector for dengue and Zika virus, has been expanding worldwide since the 20th century as climate change and international transport accelerate. It is currently identified as a major invasive species (Benedict et al., 2007; ISSG, 2009). Aedes albopictus is more heat- and stress-tolerant and is more widely distributed in Asia than Ae. aegypti (Lwande et al., 2019). In addition, this species can carry 22 more strains of arboviruses than Ae. aegypti, and is likely to be a major vector in areas where Ae. aegypti is absent (Mitcell et al., 1995; Gratz, 2004; Medlock et al., 2015).

In Korea, 12 species of mosquitoes, including Ae. albopictus, are known to transmit vector-borne diseases (Lee, 2017). Every mosquito-borne disease is managed by the Korea Disease Control and Prevention Agency (KDCA) as 'a legal infectious disease' necessitating extra surveillance because of the possibility of being introduced from abroad and exacerbated by climate change (KDCA, 2020). Although there have been no reported cases of these viruses as indigenous to Korea, a mosquito infected with dengue virus was found near the airport in Incheon, Korea in 2019 suggesting that the spread of vector-borne diseases due to introductions and climate change is a real threat (KDCA, 2019).

There are six species of the Aedini tribe (Aedes albopictus, Aedes flavopictus, Ochlerotatus japonicus, Ochlerotatus koreicus, Ochlerotatus togoi, and Ochlerotatus hatorii) living in Korea which are difficult to identify and distinguish morphologically. Aedes flavopictus, a potential vector of the dengue virus, was thought to be restricted to Korea and Japan but has recently been found in Europe (Srisawat et al., 2015; Ibáñez-Justicia et al., 2019). Ochlerotatus japonicus has recently spread to North America and Europe and is known to transmit West Nile virus, Chikungunya virus and dengue virus (Turell et al., 2001; Schaffner et al., 2011; Koban et al., 2019). Ochlerotatus koreicus is a common species that lives in urban areas, and it is a vector of Japanese encephalitis and Chikungunya virus (Shestakov and Mikheeva, 1966; Ciocchetta et al., 2018). Ochlerotatus togoi, which is the main vector of lymphatic filariasis, lives in coastal areas of east Asia and also exists along the coast of North America and South America (Cheun et al., 2011; Wada et al., 2011; Peach et al., 2019).

Successful mitigation of these mosquito-borne diseases requires regular monitoring of the range and population density of their mosquito vectors. This monitoring requires rapid and accurate methods to identify mosquito species. However, morphological identification is difficult, especially if the scales or legs which are used in identification are lost (Tanaka et al., 1979; Ree et al., 2003), and the development of a rapid and accurate identification method would be extremely useful. In this study, we describe the development of a new molecular diagnostic method for these six mosquito species using the internal transcribed spacer 2 (ITS2) of the ribosomal RNA (rRNA) region.

\section{Methods}




\section{Mosquito sample collection and information}

From August to September 2019, adults and larval mosquitoes were collected from mosquito habitats in five regions (Chiak-mountain, Yongmun-mountain, Daedeok-mountain, Bibong-mountain, and Bomokpo port) in Korea (Fig. 1). Adult specimens were collected using BG-sentinel ${ }^{T M}$ (BGS) traps (Biogents, Regensburg, Germany) with lactic acid and dry ice followed by identification using morphological criteria (Tanaka et al., 1979; Ree et al., 2003). Identified samples were stored at $-70^{\circ} \mathrm{C}$. Larvae were collected from water pools around streams and reared in the laboratory. Hatched larvae were used for the study after identification.

\section{Sequence determination of the ITS2 region of six Aedini species}

Each DNA was extracted using the AccuPrep ${ }^{\circledR}$ DNA Extraction Kit (Bioneer, K3032). Universal primers (Forward primer: 5'-AGG ACA CAT GAA CAC CCA CA-3')/ Reverse primer: 5'-CTC GCA GCT ACT CAG GGA AT-3') were designed from sequences registered in the National Center for Biotechnology Information (NCBI) with the following accession numbers: Ae. flavopictus - AF353524; Ae. albopictus - MN062758; Oc. koreicus - KF471622; Oc. togoi - EU980394; and Oc. japonicus - KF471614). The sequence of the ITS2 region of Oc. hatorii was not available as of this study, and so the sequence of the phylogenetically close Oc. togoi was used instead (Soghigian et al., 2017). The analyzed sequence data were deposited in NCBI (accessions Ae. albopictus- MT992619; Ae. flavopictus- MW040082; Oc. koreicus- MW046043; Oc. japonicus- MW046046; Oc. hatorii- MW046045; Oc. togoi- MW046044).

Individual reaction mixtures $(25 \mu \mathrm{l})$ contained: $0.4 \mu \mathrm{M}$ of each primer; $1 \mathrm{X}$ PCR buffer; $0.2 \mathrm{mM}$ of each dNTP; $1.0 \mathrm{mM} \mathrm{MgCl}_{2}$ and; 0.5 units of Taq DNA polymerase (TaKaRa, R001AM) with $1.0 \mu$ of the genomic DNA extracted from an individual specimen. Amplification was conducted on a Thermal Cycler Dice (TaKaRa, TP350) as follows: one cycle of $5 \mathrm{~min}$ at $94^{\circ} \mathrm{C}, 35$ cycles of $30 \mathrm{~s}$ at $94^{\circ} \mathrm{C}, 30 \mathrm{~s}$ at $55^{\circ} \mathrm{C}, 30 \mathrm{~s}$ at $72^{\circ} \mathrm{C}$, and final extension at $72^{\circ} \mathrm{C}$ for $10 \mathrm{~min}$. PCR products were visualized on 1.5\% (wt/vol) agarose gels stained with Ethidium Bromide (VWR life science), and then sequenced in both directions by Macrogen (Macrogen Daejeon, Korea). Sequences were aligned and analyzed using BLAST and Bioedit v7.2.6.1 (Johnson et al., 2008; Hall 1999)

\section{Multiplex PCR assay for six Aedini species}

Table 1

Universal forward primer sequences and specific reverse primer sequences for six species.

\begin{tabular}{|llll|}
\hline Species & Forward primer $\left(\mathbf{5}^{\prime} \boldsymbol{}^{\prime} \mathbf{3}^{\prime}\right)$ & Reverse primer $\left(\mathbf{5}^{\prime} \mathbf{}^{\prime} \mathbf{3}^{\prime}\right)$ & Product length (bp) \\
\hline Ae. flavopictus & AGG ACA CAT GAA CAC CCA CA & TGA GGC CTA CTG ACT TGA CTT G & 495 \\
\hline Ae. albopictus & GGA GCA CAC TGA GAG TTC CA & 438 \\
\hline Oc. koreicus & GCC TAC TGA TTG ACG GGG TA & 361 \\
\hline Oc. togoi & AGG CGG TGG AGT GTA TGG & 283 \\
Oc. hatorii & CAA TGT TTT ACC GCT GTT TGC & 220 \\
\hline Oc. japonicus & TAT ACT ACG CTG CCG AGA GG & 160 \\
\hline
\end{tabular}

Specific multiplex primer sets for the six species were designed using the same universal forward primer used for sequencing and reverse primers designed from the sequence to amplify fragments within the ITS2 region of different lengths for each species. The multiplex PCR was conducted in a $25 \mu \mathrm{l}$ reaction with $0.4 \mu \mathrm{M}$ of each primer (Table 1), $1 \mathrm{X}$ PCR buffer, $0.2 \mathrm{mM}$ of each dNTP, $1.0 \mathrm{mM} \mathrm{MgCl2,} 0.5$ units of Taq DNA polymerase (TaKaRa, R001AM) and $1.0 \mu \mathrm{l}$ of genomic DNA of an individual specimen. The PCR amplification was conducted as follows: one cycle of $5 \mathrm{~min}$ at $94^{\circ} \mathrm{C}, 35 \mathrm{cycles}$ of $30 \mathrm{~s}$ at $94^{\circ} \mathrm{C}, 30 \mathrm{~s}$ at $56^{\circ} \mathrm{C}, 30 \mathrm{~s}$ at $72^{\circ} \mathrm{C}$, and final extension step at $72^{\circ} \mathrm{C}$ for $5 \mathrm{~min}$. The products were visualized on $2.0 \%$ (wt/vol) agarose gels with Ethidium Bromide (VWR life science) and sequenced as described in Sect. 2 .

\section{Phylogenetic analysis for six Aedini species}

For phylogenetic analysis, Neighbor-joining method under the Kimura 2-parameter model was conducted using MEGA software version 6 (Tamura et al., 2013) to verify phylogenetic relationships and compare with the results of morphological identification. Bootstrapping based on the ITS2 sequence data was conducted with 1,000 replicates and genetic diversity between the species was compared using pairwise distances.

\section{Results And Discussion}

\section{Comparison of ITS2 sequence and multiplex PCR results}

In total, 109 samples of DNA extracted from individual mosquitoes were used for the study (20 of Ae. flavopictus, 24 of Ae. albopictus, 5 of Oc. japonicus, 20 of Oc. koreicus, 20 of Oc. hatorii, and 20 of Oc. togoi)

The lengths of the sequenced fragments of the ITS2 regions of the six Aedini species were: Ae. flavopictus 580 bp, Ae. albopictus 576 bp, Oc. koreicus 450 bp, Oc. togoi 451 bp, Oc. hatorii 406 bp, and Oc. japonicus 456 bp. The sequence of each fragment was aligned (using the Basic Local Alignment Search Tool BLAST) with the existing sequence registered with NCBI (accessions KF471630, MK765859, JF430391, FJ641870, KF471619, GU121103, EU980394, MN062758, KU497619, MF623839, AF353524, AF353553, and AF353551) 
Six multiplex primer sets consisting of the aforementioned universal forward primer paired with a species-specific reverse primer from within the ITS2 region were designed to amplify fragments of different species-specific lengths (Fig. 2). A gel showing the results of multiplex analysis of DNA from each species is shown in Fig. 3 (Ae. flavopictus 495 bp, Ae. albopictus 438 bp, Oc. korecus 361 bp, Oc. togoi 283 bp, Oc. hatorii 220 bp, Oc. japonicus 160 bp).

\section{Results of phylogenetic analysis using Mega 6}

The ITS2 sequences of the six Aedini species used in this study and other ITS2 sequences registered in NCBI from other species in the Aedini tribe (accessions KF471630, MK765859, JF430391, FJ641870, KF471619, GU121103, EU980394, MN062758, KU497619, MF623839, AF353524, AF353553, and AF353551) were analyzed to determine phylogenetic relatedness (Fig. 4). Aedes albopictus and Ae. flavopictus which are morphologically similar were closely related as were Oc. koreicus and Oc. japonicus. The phylogenetic tree was clearly divided into Aedes sp. and Ochlerotatus sp., but Oc. hatorii and Oc. togoi were only distantly related to the other Ochlerotatus sp. A pairwise analysis of genetic distances using the Kimura 2-parameter calculation and the interspecies ITS2 region showed $35.0 \pm 1.5 \%$ difference between Aedes spp. and Ochlerotatus spp., $17.4 \pm 0.2 \%$ difference between Ae. albopictus and Ae. flavopictus, and 11.1 $\pm 0.3 \%$ difference between Oc. koreicus and Oc. japonicus (Table 2). Although the species were morphologically indistinguishable, the phylogenetic relationship between six species of Aedini tribe as well as between the genera was confirmed through this progress.

Table 2

An analysis of pairwise distances using the Kimura 2-parameter calculation for six Aedin

\begin{tabular}{|c|c|c|c|c|c|c|c|c|c|c|c|c|c|}
\hline Accession & $\mathrm{DQ}$ & MT & MN & MF & $\mathrm{KU}$ & $\mathrm{AF}$ & $\mathrm{AF}$ & $\mathrm{AF}$ & MW & MW & MK & JF & KF \\
\hline number & 341113 & 992619 & 062758 & 623839 & 497619 & 353524 & 353551 & 353553 & 040082 & 046043 & 765859 & 430391 & 471630 \\
\hline DQ341113 & - & & & & & & & & & & & & \\
\hline MT992619 & $36.44 \%$ & - & & & & & & & & & & & \\
\hline MN062758 & $36.23 \%$ & $0.17 \%$ & - & & & & & & & & & & \\
\hline MF623839 & $36.11 \%$ & $0.35 \%$ & $0.17 \%$ & - & & & & & & & & & \\
\hline KU497619 & $36.31 \%$ & $0.70 \%$ & $0.52 \%$ & $0.70 \%$ & - & & & & & & & & \\
\hline AF353524 & $36.67 \%$ & $17.59 \%$ & $17.41 \%$ & $17.27 \%$ & $17.63 \%$ & - & & & & & & & \\
\hline AF353551 & $36.67 \%$ & $17.83 \%$ & $17.65 \%$ & $17.32 \%$ & $17.86 \%$ & $0.00 \%$ & - & & & & & & \\
\hline AF353553 & $36.47 \%$ & $17.41 \%$ & $17.24 \%$ & $17.09 \%$ & $17.45 \%$ & $0.69 \%$ & $0.69 \%$ & - & & & & & \\
\hline MW040082 & $36.75 \%$ & $17.27 \%$ & $17.45 \%$ & $17.30 \%$ & $17.66 \%$ & $0.17 \%$ & $0.17 \%$ & $0.70 \%$ & - & & & & \\
\hline MW046043 & $35.56 \%$ & $34.77 \%$ & $35.00 \%$ & $35.00 \%$ & $35.45 \%$ & $34.75 \%$ & $34.75 \%$ & $34.75 \%$ & $34.61 \%$ & - & & & \\
\hline MK765859 & $36.12 \%$ & $36.26 \%$ & $36.04 \%$ & $36.04 \%$ & $36.26 \%$ & $35.41 \%$ & $35.41 \%$ & $35.41 \%$ & $35.71 \%$ & $1.56 \%$ & - & & \\
\hline JF430391 & $36.12 \%$ & $36.26 \%$ & $36.04 \%$ & $36.04 \%$ & $36.26 \%$ & $35.41 \%$ & $35.41 \%$ & $35.41 \%$ & $35.71 \%$ & $1.56 \%$ & $0.00 \%$ & - & \\
\hline KF471630 & $35.56 \%$ & $35.23 \%$ & $35.00 \%$ & $35.00 \%$ & $35.45 \%$ & $34.75 \%$ & $34.75 \%$ & $34.75 \%$ & $35.06 \%$ & $0.44 \%$ & $1.56 \%$ & $1.56 \%$ & - \\
\hline MW046046 & $35.75 \%$ & $34.38 \%$ & $34.60 \%$ & $34.68 \%$ & $35.12 \%$ & $35.48 \%$ & $35.48 \%$ & $35.48 \%$ & $35.33 \%$ & $10.63 \%$ & $10.99 \%$ & $10.99 \%$ & $11.09 \%$ \\
\hline KF471619 & $35.67 \%$ & $34.74 \%$ & $34.52 \%$ & $34.60 \%$ & $34.82 \%$ & $35.18 \%$ & $35.18 \%$ & $35.18 \%$ & $35.48 \%$ & $11.06 \%$ & $10.51 \%$ & $10.51 \%$ & $11.06 \%$ \\
\hline FJ641870 & $35.96 \%$ & $34.82 \%$ & $34.60 \%$ & $34.68 \%$ & $35.12 \%$ & $35.48 \%$ & $35.48 \%$ & $35.48 \%$ & $35.78 \%$ & $11.54 \%$ & $11.43 \%$ & $11.43 \%$ & $11.54 \%$ \\
\hline GU121103 & $36.17 \%$ & $34.81 \%$ & $34.59 \%$ & $34.67 \%$ & $34.89 \%$ & $35.24 \%$ & $35.24 \%$ & $35.24 \%$ & $35.54 \%$ & $11.49 \%$ & $10.94 \%$ & $10.94 \%$ & $11.49 \%$ \\
\hline MW046045 & $34.24 \%$ & $31.17 \%$ & $31.42 \%$ & $31.42 \%$ & $31.92 \%$ & $30.42 \%$ & $30.42 \%$ & $30.42 \%$ & $30.25 \%$ & $11.28 \%$ & $11.25 \%$ & $11.25 \%$ & $11.78 \%$ \\
\hline MW046044 & $40.31 \%$ & $34.54 \%$ & $34.76 \%$ & $34.62 \%$ & $34.84 \%$ & $37.67 \%$ & $37.67 \%$ & $37.44 \%$ & $37.53 \%$ & $19.16 \%$ & $19.71 \%$ & $19.71 \%$ & $19.66 \%$ \\
\hline EU980394 & $40.00 \%$ & $34.68 \%$ & $34.46 \%$ & $34.31 \%$ & $34.54 \%$ & $37.36 \%$ & $37.36 \%$ & $37.14 \%$ & $37.67 \%$ & $19.12 \%$ & $19.17 \%$ & $19.17 \%$ & $19.12 \%$ \\
\hline
\end{tabular}

Out group for Culex pipiens pallens: DQ341113. Ae. albopictus: MT992619, MN062758, MF623839, KU497619. Ae. flavopictus: MW040082, AF353524, AF35 KF471630. Oc. japonicus: MW046046, KF471619, FJ641870, GU121103. Oc. togoi: MW046044, EU980394. Oc. hatorii: MW046045.

\section{Application of multiplex PCR molecular diagnostic method}

All of the species in this study were very similar morphologically, and were identified by fine differences in the leg, scales, scutum and scutellum (Tanaka et al., 1979; Ree et al., 2003).

The tediousness of accurate morphological identification and the fact that legs and scales may be lost during collection or storage results in a substantial mis-identification rate. The multiplex PCR assay we developed can reduce this mis-identification rate and is simple - requiring only PCR followed by electrophoresis. A multiplex PCR assay for Anopheles sp. which transmit malaria has also been developed and is being used to monitor certain vector species of Anopheles sp. (Scott et al., 1993, Joshi et al., 2010). 
The multiplex PCR assay we developed for Aedini species enables simple and accurate identification and monitoring of species of mosquitoes that carry flaviviruses such as dengue virus, yellow fever virus, and Zika virus. The data provided by consistent and accurate monitoring of mosquito populations through this method can potentially be used to guide national public health measures, such as quarantining and thus help prevent the spread of infectious mosquito-borne diseases.

\section{Conclusions}

In this study, a multiplex PCR assay was developed to identify six Aedini species which can transmit various diseases in Korea. The resulting assay provides a simple and accurate molecular identification tool for these six species, which are difficult to identify morphologically. These species are expected to spread globally due to climate change and increasing international trade. This tool will, therefore, be useful for control of the vectors for several infectious diseases.

\section{Declarations}

\section{Availability of data and materials}

The datasets generated during the current study are included within the article. Sequences used in this study are deposited in NCBI GenBank database (https://www.ncbi.nlm.nih.gov/genbank/) under the accession numbers MT992619 MW040082 MW046043 MW046046 MW046045 MW046044.

\section{Author's contributions}

WJB designed the study, developed the new multiplex PCR assay method for six Aedini species and drafted the manuscript. MHW and STC assisted with collecting mosquitoes and identifying mosquito species. JR designed the study with WJB, and assisted with analysis of the data and helped draft the manuscript. KSC revised and improved the manuscript. All authors approved the final manuscript.

\section{Acknowledgements}

This research was supported by Basic Science Research Program through the National Research Foundation of Korea (NRF) funded by the Ministry of Education (2020R1T1A2066186).

\section{Funding}

This research was supported by Basic Science Research Program through the National Research Foundation of Korea (NRF) funded by the Ministry of Education (2020R1T1A2066186).

\section{Ethics declarations}

\section{Ethics approval and consent to participate}

Not applicable.

\section{Consent for publication}

Not applicable.

\section{Competing interests}

The authors declare that they have no competing interests.

\section{References}

1. Attaway DF, Waters NM, Geraghty EM, Jacobsen KH. Zika virus: Endemic and epidemic ranges of Aedes mosquito transmission. J Infect Public Health. 2017;10:120-123.

2. Benedict MQ, Levine RS, Hawley WA, Lounibos LP. Spread of the Tiger. Vector Borne Zoonotic Dis. 2007;7:76-85.

3. Bhatt S, Gething PW, Brady OJ, Messina JP, Farlow AW, Moyes CL, et al. The global distribution and burden of dengue. Nature. 2013;496:504-507.

4. Cheun H II, Cho SH, Lee H II, Shin EH, Lee JS, Kim TS, et al. Seasonal Prevalence of Mosquitoes, Including Vectors of Brugian Filariasis, in Southern Islands of the Republic of Korea. Korean J Parasitol. 2011;49:59-64.

5. Ciocchetta S, Prow NA, Darbro JM, Frentiu FD, Savino S, Montarsi F, et al. The new European invader Aedes (Finlaya) koreicus: a potential vector of chikungunya virus. Pathog Glob Health. 2018;112:107-114.

6. Gratz NG. Critical review of the vector status of Aedes albopictus. Med Vet Entomol. 2004;18:215-27.

7. Hall, T.A. BioEdit: A User-Friendly Biological Sequence Alignment Editor and Analysis Program for Windows 95/98/NT. Nucleic Acids Symposium Series. 1999;41:95-98.

8. Ibáñez-Justicia A, van de Vossenberg B, van den Biggelaar R, Voogd J, et al. Detection of Aedes flavopictus (Yamada, 1921), Netherlands, June 2019. Euro Surveill. 2019;24(30):1900433. 
9. ISSG. Global Invasive Species Database- Aedes albopictus. The Invasive Species Specialist Group. 2009; Available at http://www.issg.org/database/species/ecology.asp?si=109\&fr=1\&sts=sss\&lang=EN. Accessed December 7, 2020).

10. Joshi D, Park MH, Saeung A, Choochote W, Min GS. Multiplex assay to identify Korean vectors of malaria. Mol Ecol Resour. 2010;10:748-750.

11. Johnson M, Zaretskaya I, Raytselis Y, Merezhuk Y, et al. NCBI BLAST: a better web interface. Nucleic Acids Res. 2008;36:5-9

12. KDCA. Dengue virus detection through mosquito surveillance in Incheon, Republic of Korea. Korea Disease Control and Prevention Agency. 2019; Available at http://www.kdca.go.kr/board.es?mid=a20501000000\&bid=0015\&act=view\&list_no=364502. Accessed December 7, 2020).

13. KDCA. Infectious Disease Portal. Korea Disease Control and Prevention Agency. 2020; Available at http://www.kdca.go.kr/npt/biz/npp/nppMain.do. Accessed December 7, 2020.

14. Koban, M.B., Kampen, H., Scheuch, D.E. et al. The Asian bush mosquito Aedes japonicus japonicus (Diptera: Culicidae) in Europe, 17 years after its first detection, with a focus on monitoring methods. Parasites Vectors 2019;12:109

15. Lee DK. Ecological characteristics and current status of infectious disease vectors in South Korea. J Korean Med Assoc. 2017;60:458-467.

16. Lwande OW, Obanda V, Lindström A, AhIm C, Evander M, Näslund J, et al. Globe-Trotting Aedes aegypti and Aedes albopictus: Risk Factors for Arbovirus Pandemics. Vector-Borne Zoonotic Dis. 2020;20:71-81.

17. Medlock JM, Hansford KM, Versteirt V, Cull B, Kampen H, Fontenille D, et al. An entomological review of invasive mosquitoes in Europe. Bull Entomol Res. 2015;105:637-663.

18. Mitchell C. Geographic spread of Aedes albopictus and potential for involvement in arbovirus cycles in the Mediterranean basin. J Vector Ecol 1995; 20:44-58.

19. Peach DAH, Almond M, Pol JC. Modeled distributions of Aedes japonicus japonicus and Aedes togoi (Diptera: Culicidae) in the United States, Canada, and northern Latin America. J Vector Ecol. 2019;44(1):119-129.

20. REE H-I. Taxonomic Review and Revised Keys of the Korean Mosquitoes (Diptera: Culicidae). Entomol Res. 2003;33:39-52.

21. Reither P. Climate Change and mosquito-borne disease. Environ Int. 2001;109:141-161

22. Schaffner F, Vazeille M, Kaufmann C, Failloux A-B, Mathis A. Vector competence of Aedes japonicus for chikungunya and dengue viruses. J Eur Mosq Control Assoc. 2011;29:141-2.

23. Scott JA, Brogdon WG, Collins FH. Identification of single specimens of the Anopheles gambiae complex by the polymerase chain reaction. Am J Trop Med Hyg. 1993;49(4):520-529.

24. Shestakov VI, Mikheeva Al. K. Study of vectors of Japanese encephalitis in the Maritime Territory. Med Parazitol. 1966;35(5):545-550.

25. Soghigian J, Andreadis TG, Livdahl TP. From ground pools to treeholes: Convergent evolution of habitat and phenotype in Aedes mosquitoes. BMC Evol Biol;2017;17:1-13.

26. Srisawat R, Phanitchat T, Komalamisra N, Tamori N, Runtuwene L, Noguchi K, et al. Susceptibility of Aedes flavopictus miyarai and Aedes galloisi mosquito species in Japan to dengue type 2 virus. Asian Pac J Trop Biomed 2016;6:446-450.

27. Tanaka K, Mizusawa K, Saugstad ES, et al. A Revision of the adult and larval mosquitoes of Japan (including the Ryukyu Archipelago and the Ogasawara islands) and Korea (Diptera: Culicidae), Contrib Am Entomol Inst, U.S.A. 1979;16

28. Tatem AJ, Huang Z, Das A, Qi Q, Roth J, Qiu Y. Air travel and vector-borne disease movement. Parasitology. 2012;139:1816-1830.

29. Tamura K, Stecher G, Peterson D, Filipski A, et al. MEGA6: Molecular Evolutionary Genetics Analysis version 6.0. Mol Biol Evol. 2013;30(12):2725-2729.

30. Turell MJ, O'Guinn ML, Dohm DJ, Jones JW. Vector Competence of North American Mosquitoes (Diptera: Culicidae) for West Nile Virus. J Med Entomol. 2001;38:130-134.

31. Wada Y. Vector mosquitoes of filariasis in Japan. Trop Med Health. 2011;39:39-45.

32. WHO. Vector-borne diseases. World Health Organization. 2020; Available at: https://www.who.int/en/news-room/fact-sheets/detail/vector-borne-diseases. Accessed December 07, 2020.

\section{Figures}




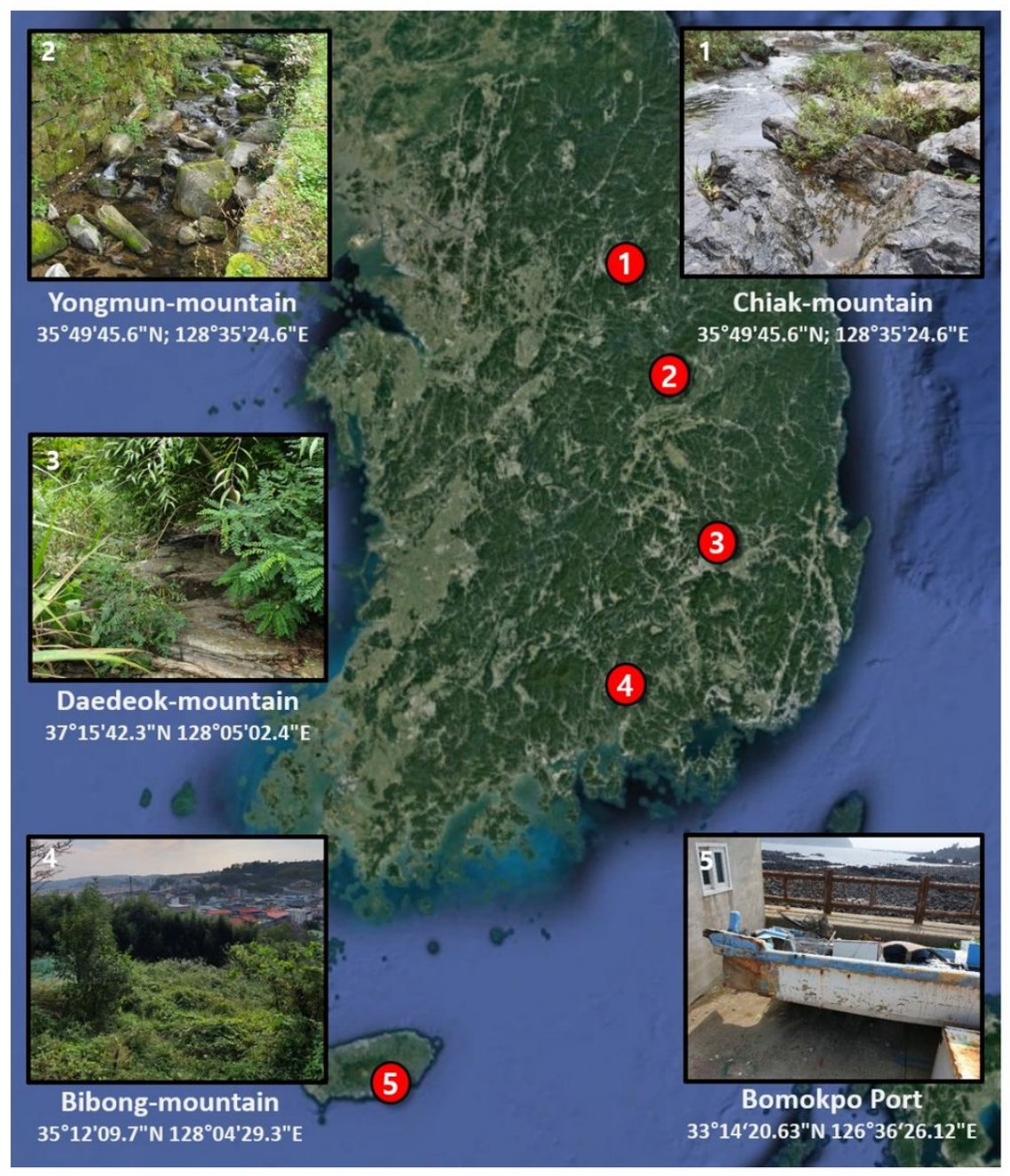

\section{Figure 1}

Collection sites of the six Aedini species in Korea. 1: Chiak mountain. 2: Yongmun-mountain. 3: Daedeok-mountain. 4: Bibong-mountain. 5: Bomokpo port. Note: The designations employed and the presentation of the material on this map do not imply the expression of any opinion whatsoever on the part of Research Square concerning the legal status of any country, territory, city or area or of its authorities, or concerning the delimitation of its frontiers or boundaries. This map has been provided by the authors.

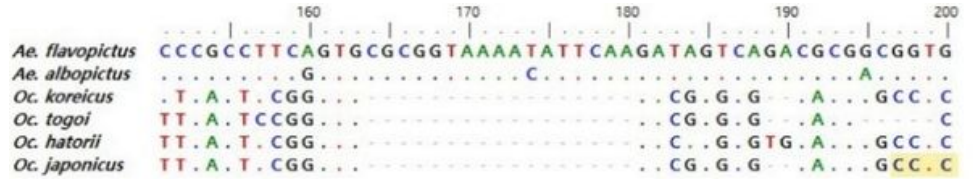

$$
210
$$

220

230

\begin{abstract}
240
\end{abstract}
250

Ae. flavopictus GCCGGCG TGCCAG T CG T CG T CG TGG T TGA TGAGTACA T CCCAA- C CGGAG Ae albopictus
$\begin{aligned} & \text { OC. koreicus } \\ & \text { OC. togoi }\end{aligned}$

Oc. togoi

Oc. hatorii CA. ACA.AA C...A.A.T...ACTC.........GG.C

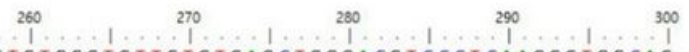

Ae. flavopictus TGTGTG TGTGTGGGTGTTGTGTGAGCTGGGACG T C CTCAAGGGTGGCAG

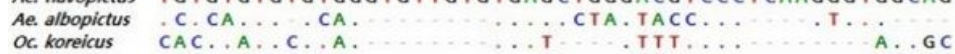

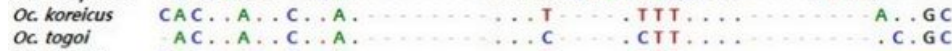
Oc. hatorii CAC.......... A.

Oc. japonicus CAC. A. C...

$$
310 \quad 320 \quad 330 \quad 340
$$

Ae. flavopictus TGGACTGCGCGTTCCCTCTCCGTGGAATT TGGCGCGTGCGCGGGAGCGCA

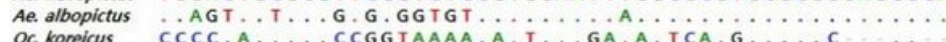

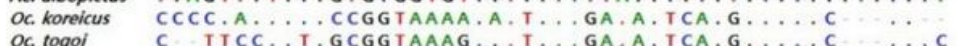
Oc. hatorii

Oc. japonicus

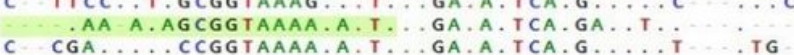

\section{Figure 2}

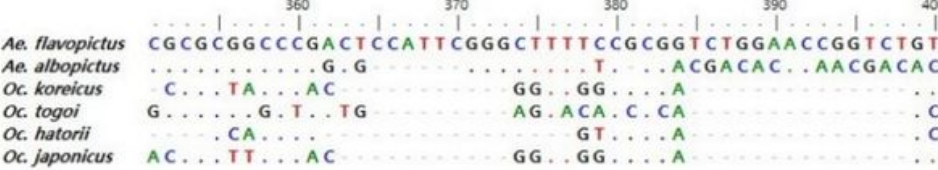

$$
410
$$$$
420 \quad 430
$$

$130 \quad 440 \quad 1 \ldots 1 \ldots$ Ae. albopictus CKG TCACCCGG TCCTGGCAAGCTCTAAGTACAAACGCCGCC ACA C C C

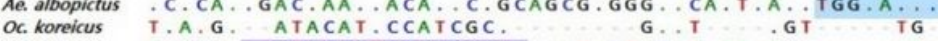

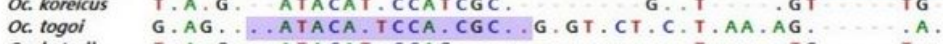

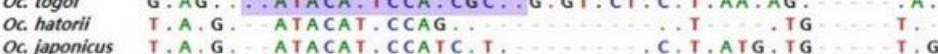
Oc. japonicus T.A.G. ATACA T. CCA TC.T.

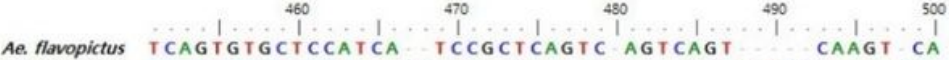
Ae. albopictus

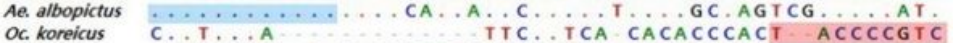

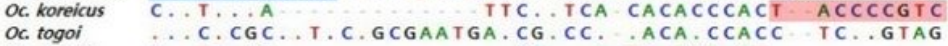
Oc. japonicus C. TC.GC . CA...A TR,AGA,AT

$\begin{array}{llll}510 & 520 & 530 & 540\end{array}$ Ae. flavopictus AGTCAGTAGGCCTCAAATAATGTGTGACTACCCCCTAAATTTAAGCATAT

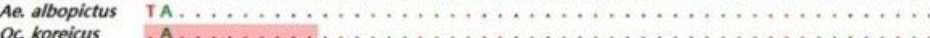

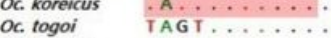

Oc. hatorii

Oc. japonicus 
Position of six specific reverse primers within the ITS2 region.

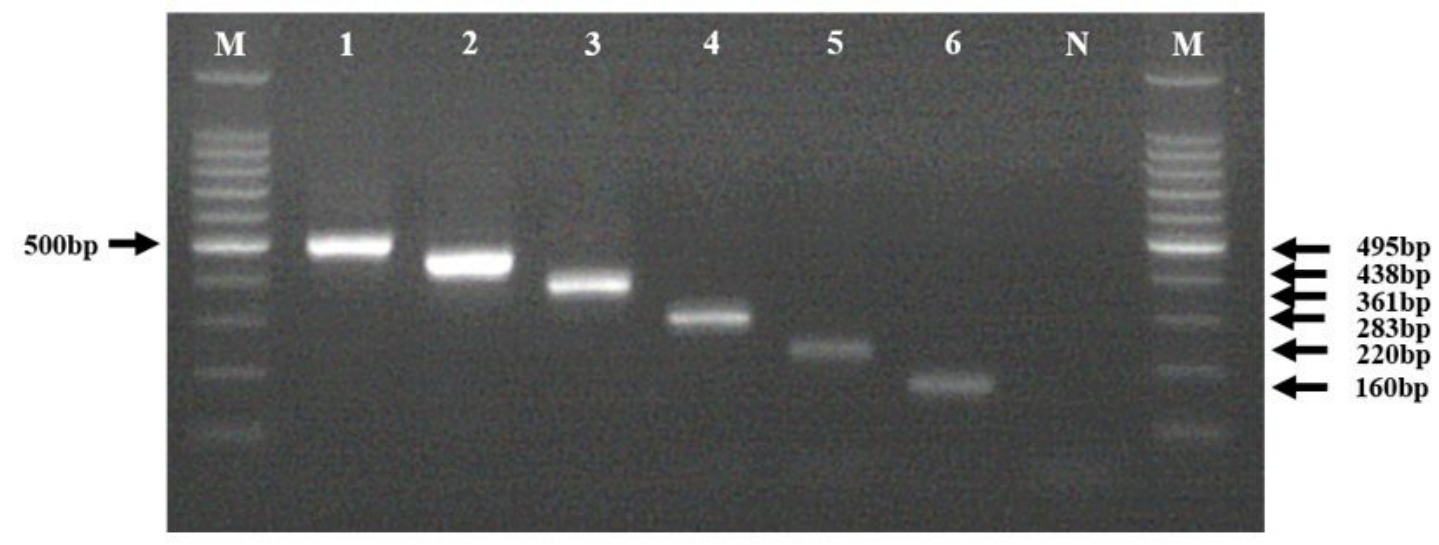

\section{Figure 3}

A result of multiplex PCR assay for six Aedini species. M: 100bp molecular marker; lane 1: Ae. flavopictus; lane 2: Ae. albopictus; land 3: Oc. koreicus; lane 4: Oc. togoi; lane 5: Oc. hatorii; lane 6: Oc. japonicus.

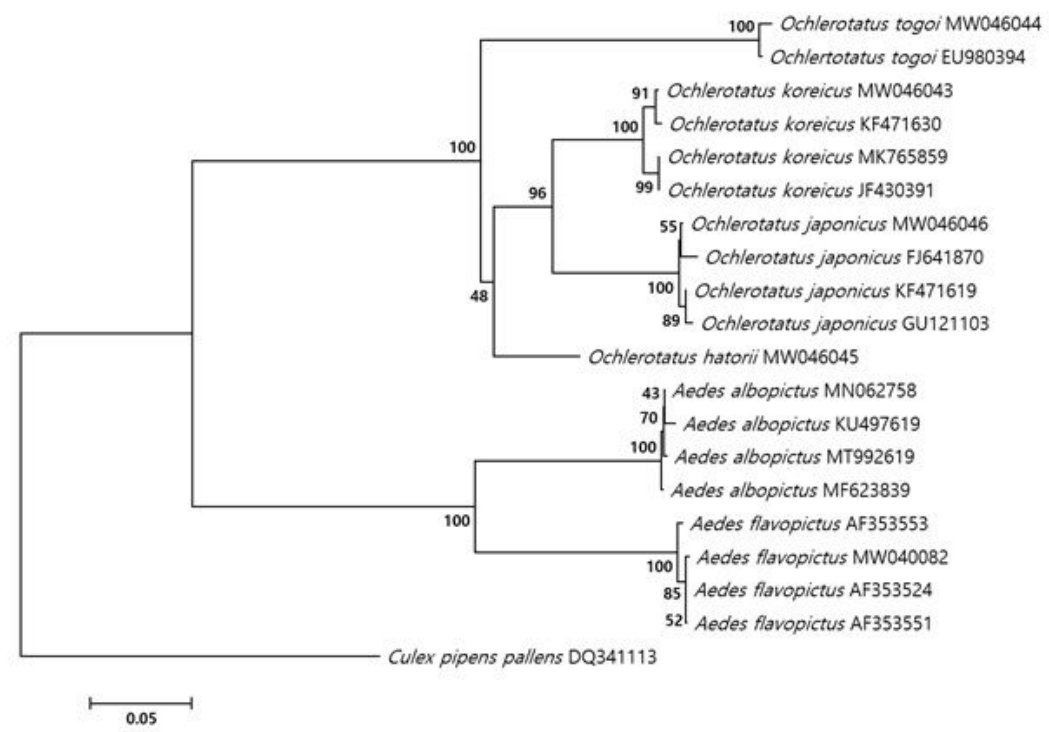

\section{Figure 4}

Neighbor joining phylogenetic tree (Kimura 2-parameter genetic distance calculating method) using ITS2 sequences of six species.

\section{Supplementary Files}

This is a list of supplementary files associated with this preprint. Click to download.

- WooJunBanggraphicalabstractsize.jpg 